\title{
REPORTE DE CASO DE RESISTENCIA AL GANCICLOVIR EN ENFERMEDAD POR CITOMEGALOVIRUS POSTRASPLANTE CARDIACO
}

\author{
Christian Rojas-Contreras ${ }^{1, a}$, Gabriel De la Cruz-Kü,b, Stalin Vilcarromero ${ }^{3, a}$, Ruben Villacaqui Ayllon ${ }^{4, c}$ \\ Bryan Valcarcel-Valdivia2,b
}

\begin{abstract}
RESUMEN
La infección por citomegalovirus postrasplante cardiaco es una condición médica recurrente. Su frecuencia se incrementa cuando los donantes poseen serología positiva y los receptores presentan serología negativa para el virus. En la población pediátrica, la enfermedad solo se desarrolla en un porcentaje pequeño y raramente presentan resistencia al tratamiento convencional con ganciclovir y valganciclovir. Presentamos el primer reporte de caso pediátrico de enfermedad por citomegalovirus resistente a ganciclovir y valganciclovir postrasplante cardiaco en un hospital público peruano, con una presentación inusual. La resistencia a estos fármacos fue evidente luego de 277 días de evolución de la enfermedad, ante la no remisión de la sintomatología y la persistencia de una carga viral elevada. La posterior administración de foscarnet condujo a una mejora clínica y de laboratorio, hasta la remisión de la enfermedad.
\end{abstract}

Palabras clave: Trasplante cardiaco; Pediatría; Virosis; Resistencia antiviral; Foscarnet, Perú (fuente: DeCS BIREME).

\section{RESISTANCE TO GANCICLOVIR IN CYTOMEGALOVIRUS INFECTION AFTER HEART TRANSPLANT: CASE REPORT}

\begin{abstract}
Cytomegalovirus infection after a heart transplant is a recurrent medical condition. Its frequency increases when the donors are serum-positive, and the recipients are serum-negative to this virus. In the pediatric population, the infection only develops in a small percentage and the patients rarely present resistance to conventional treatment with ganciclovir and valganciclovir. We presented the first report of a pediatric case of the cytomegalovirus infection resistant to ganciclovir and valganciclovir after a heart transplant in a Peruvian public hospital with an unusual presentation. The resistance to these drugs was evident after 277 days of evolution of the disease considering the non-remission of the symptomatology and the persistence of an elevated viral load. The administration of foscarnet led to a clinical and laboratory improvement until remission of the disease.
\end{abstract}

Keywords: Heart transplantation; Pediatría; Virus diseases; Antiviral resistance; Foscarnet, Peru (source: MeSH NLM).

\section{INTRODUCCIÓN}

La infección por citomegalovirus (CMV) genera una condición de alta morbilidad y mortalidad en los pacientes postrasplantados de corazón ${ }^{(1)}$. En la población pediátrica, la enfermedad por CMV (ECMV), definida como la infección sintomática por CMV se desarrolla aproximadamente entre el $11-18 \%$. La ECMV resistente a ganciclovir (ECMV-RG) ocurre en menos del $2 \%$ de la población pediátrica, con mal pronóstico clínico. Además, su incidencia es del $5 \%$ entre los pacientes de alto riesgo, definidos como donante positivo y receptor negativo ( $\mathrm{D}+/ \mathrm{R}-)$ para la infección por $\mathrm{CMV}$ y el $12 \%$ entre los que desarrollan ECMV postrasplante cardiaco ${ }^{(1-4)}$.

Según la Sociedad Internacional de Trasplante Cardíaco y Pulmonar (ISHLT, por sus siglas en inglés), la terapia profiláctica incluye 3-6 meses de ganciclovir intravenoso, seguido de valganciclovir oral. El tratamiento de la ECMV es con los mismos fármacos ${ }^{(5)}$. No obstante, la resistencia a estos fármacos, definida como un fracaso clínico sin mejoría después de 14-21 días de tratamiento,

Servicio de Infectología, Instituto Nacional Cardiovascular. Lima, Perú

Sociedad científica de estudiantes de medicina, Universidad Científica del Sur (UCSUR). Lima, Perú

Naval Medical Research Unit No 6. Iquitos, Perú

Universidad Científica del Sur (UCSUR). Lima, Perú

Médico infectologo; ${ }^{\mathrm{b}}$ estudiante de Medicina; ${ }^{\mathrm{c}}$ médico veterinario

Recibido: 07/09/2017 Aprobado: 22/11/2017 En línea: 23/03/2018

Citar como: Rojas-Contreras C, De la Cruz-Ku G, Vilcarromero S, Villacaqui Ayllon R, Valcarcel-Valdivia B. Reporte de caso de resistencia al ganciclovir en enfermedad por citomegalovirus postrasplante cardiaco. Rev Peru Med Exp Salud Publica. 2018;35(1):145-9. doi: 10.17843/rpmesp.2018.351.3562. 
condiciona al uso de foscarnet ${ }^{(1,6)}$. En Perú, no hay reportes de resistencia al ganciclovir o valganciclovir postrasplante cardiaco. Además, la resistencia a estos fármacos se identifica en etapas tempranas, usualmente antes del mes ${ }^{(1,6)}$.

Se presenta el caso de un paciente pediátrico de alto riesgo, infectado con CMV tras un trasplante cardiaco. Luego de recibir los fármacos convencionales en dos ocasiones y con resolución clínica favorable, el paciente desarrolla resistencia luego de 277 días de tratamiento. Se decide iniciar la terapia con foscarnet, con lo cual la enfermedad remite. Se resalta la peculiaridad de generar resistencia a la terapia convencional después de un periodo largo de tratamiento.

\section{REPORTE DE CASO}

Se trata de un niño mestizo de 12 años de edad, proveniente de área metropolitana, con antecedente de asma y cuatro hospitalizaciones previas por esta causa. Se le realizó un trasplante cardiaco ortotópico, debido a los diagnósticos de cardiomiopatía congénita dilatada refractaria, insuficiencia cardiaca, insuficiencia mitral y tricuspídea. Previo a la cirugía, se efectuó terapia de inducción con basiliximab y corticoides (inicialmente con metilprednisolona y posteriormente con prednisona). El paciente se encontraba sin infección por CMV previo al trasplante; sin embargo, el donante presentaba la infección. Por ello, el estado serológico del paciente era de alto riesgo (D+/R-). La cirugía empleó la técnica bicaval y duró cinco horas y 50 minutos. Una ecocardiografía posoperatoria reveló una mejora en la fracción de eyección ventricular, de 25 a 65\%.

La terapia inmunosupresora postrasplante consistió en tracolimus, prednisona y micofenolato de mofetilo. La terapia profiláctica incluyó: terapia antiviral con ganciclovir (5 mg/kg/12h) por 15 días, seguido de valganciclovir vía oral (15 mg/kg/24h, un total de $450 \mathrm{mg} / 24 \mathrm{~h}$ ) por 90 días; terapia antibacterial con cefepime y trimetropin/sulfametoxazol por 6 y 90 días, respectivamente.

Luego de 121 días posterior a la cirugía, el paciente ingresó a emergencia por presentar fiebre $\left(38^{\circ} \mathrm{C}\right)$, hiporexia, dolor abdominal difuso y diarrea líquida sin moco o sangre. Las funciones vitales fueron las siguientes: presión arterial de 100/78 mmHg, latidos cardiacos de 115 por minuto y frecuencia respiratoria de 21 por minuto, saturación de oxígeno de $98 \%$ y $30 \mathrm{~kg}$ de peso corporal. El examen físico mostró mucosas orales deshidratadas, llenado capilar de dos segundos, ruidos hidroaéreos presentes, y abdomen doloroso a la palpación superficial y profunda principalmente en mesogastrio, sin signos de irritación peritoneal. Los exámenes de laboratorio mostraron neutropenia $\left(1,78 \times 10^{3} / \mathrm{mm}^{3}\right.$, valores normales $(\mathrm{VN})$ : $1,8-$ $8 \times 10^{3} / \mathrm{mm}^{3}$ ) y transaminasas elevadas (transaminasa pirúvica: 192 U/L VN: 6-38 U/L, transaminasa oxaloacética: 258 U/L VN: 9-46 U/L). Inicialmente se sospechó de infección bacteriana o viral. No obstante, una elevación de la carga viral confirmó el diagnóstico de ECMV. Se reinició tratamiento con ganciclovir intravenoso $(5 \mathrm{mg} / \mathrm{kg} / 12 \mathrm{~h})$ por 14 días. Dado la mejoría clínica, el paciente fue dado de alta con tratamiento con valganciclovir vía oral $(450 \mathrm{mg} / 24$ horas) y sin monitorización de la carga viral.

Diecinueve días después (157 días postrasplante) el paciente acudió a sus controles médicos. El examen físico fue positivo solamente para úlceras en la boca. No se evidenció alteración en las funciones vitales o en el examen físico. Los exámenes de laboratorio mostraron bicitopenia (hemoglobina: $8,3 \mathrm{mg} / \mathrm{dL}$, leucocitos: 2,62 × 103/ $\mathrm{mm}^{3}$ ), conteo de linfocitos CD4+ bajo (138 cel/lL) y carga viral alta (399 704 copias $/ \mathrm{mL}$ ) a pesar del tratamiento con valganciclovir. Se reinició el tratamiento con ganciclovir a dosis de $180 \mathrm{mg} / 12$ horas durante 40 días y $200 \mathrm{mg} / 12$ horas durante 7 días, se administró filgastrim intravenoso y tracolimus. Los niveles séricos de tracolimus siempre estuvieron dentro de los valores normales, con un promedio de $8 \mathrm{ng} / \mathrm{mL}$ (VN 5-20 ng/mL). Debido a la mejora clínica y a una viremia inferior a 200 copias $/ \mathrm{mL}$, se continuó con valganciclovir $(450 \mathrm{mg} / 12 \mathrm{~h})$ y se procedió al alta del paciente el día 215 postrasplante cardiaco (Figura 1).

En el día 277 postrasplante, el paciente fue ingresado por tercera vez debido a un cuadro diarreico agudo. Las funciones vitales fueron: temperatura corporal de $36,9^{\circ} \mathrm{C}$, frecuencia cardíaca de 109 latidos por minuto, presión arterial de 105/75 mmHg y saturación de oxígeno de $99 \%$. El examen clínico reveló dolor a la palpación superficial y profunda en epigastrio. Las pruebas de laboratorio demostraron pancitopenia (hemoglobina: 5,6 mg/dl, leucocitos: $0,97 \times 10^{3} / \mathrm{mm}^{3}$, plaquetas: 148000$)$ y una carga viral elevada (39 700 copias/ml). Se le realizó una biopsia de médula ósea mediante inmunohistoquímica para descartar alguna infección sobreagregada, donde solamente se identificó la presencia de CMV. Inicialmente se indicó ganciclovir a dosis de $7,5 \mathrm{mg} / \mathrm{kg}$, no obstante, ante la falta de mejoría clínica, la dosis se incrementó a $10 \mathrm{mg} / \mathrm{kg}$. Después de 100 días de tratamiento, la condición del paciente empeoró, con trombocitopenia marcada (95 000), linfopenia $\left(0,37 \times 10^{3} / \mathrm{mm}^{3}\right)$ y viremia de 56500 copias $/ \mathrm{mL}$. Debido a la falta de mejoría clínica y virológica, y por sospecha de resistencia al tratamiento, se decidió iniciar terapia con foscarnet 2 g/8horas en el día 379 postrasplante cardiaco (Figura 2). 


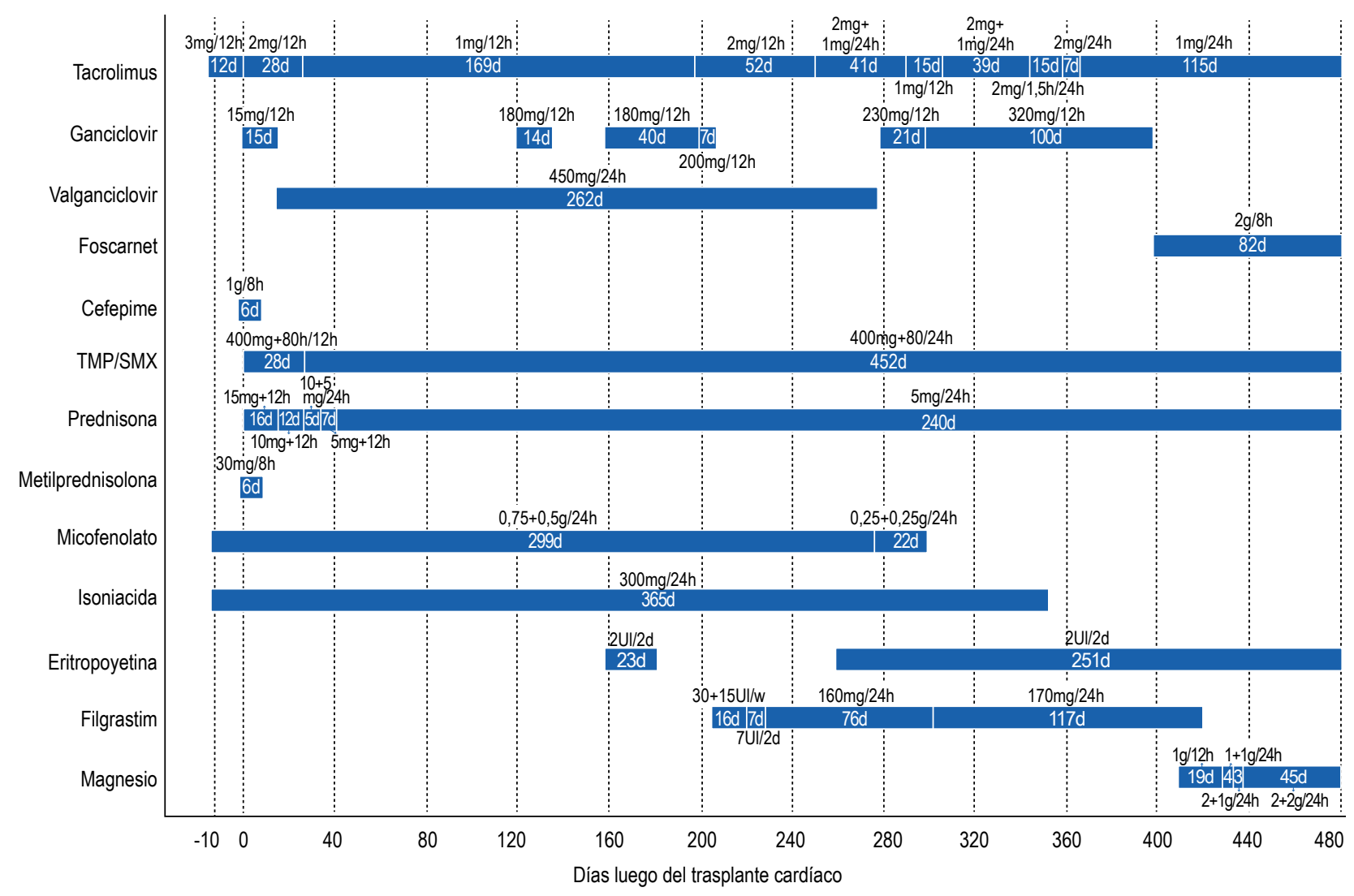

Figura 1. Lista de fármacos con sus respectivas dosis administradas al paciente de acuerdo con los días posteriores al trasplante cardíaco.

En el día 458 postrasplante cardiaco, en tratamiento con foscarnet, las cargas virales se tornaron indetectables y la condición clínica mejoró. Se continuó con la administración de foscarnet durante 90 días y se procedió al alta médica del paciente. Posteriormente, se realizaron controles mensuales y detección de la carga viral en dos oportunidades, ambos con resultados indetectables.

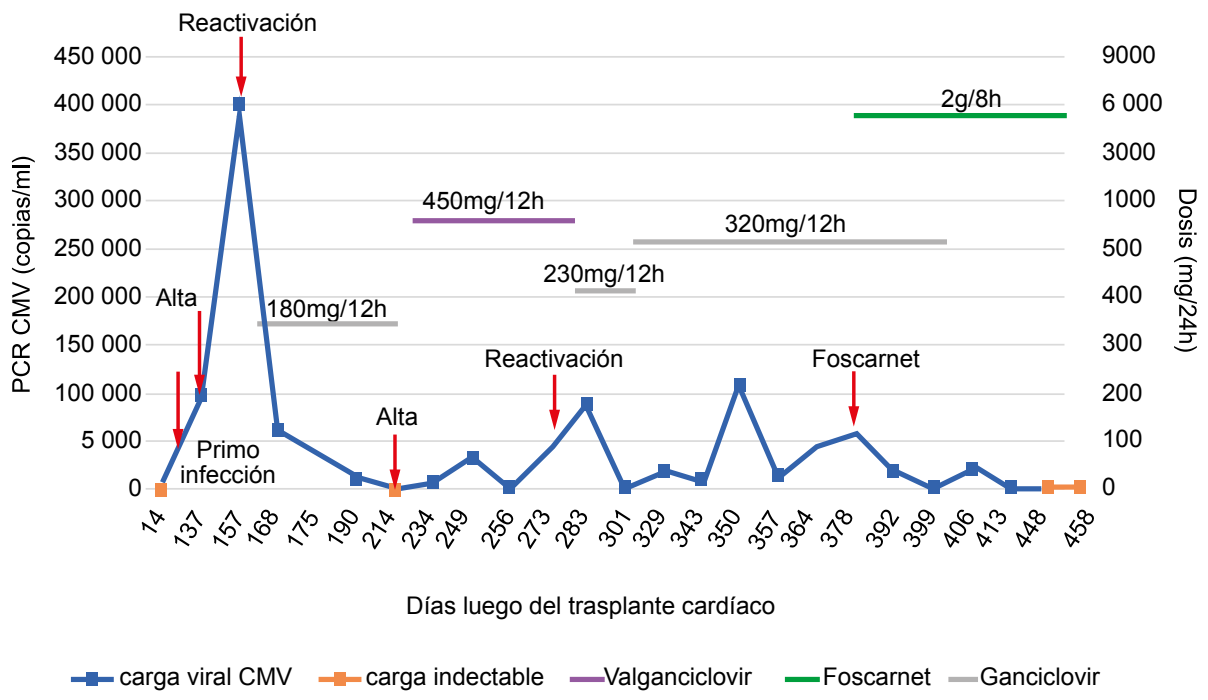

Figura 2. Evolución de la viremia según los días posteriores al trasplante cardíaco. Los fármacos terapéuticos también se presentan, de acuerdo con la historia de la enfermedad. 


\section{DISCUSIÓN}

Lainfección por CMV persiste como una importante condición de morbilidad en los pacientes postrasplante cardiaco $(2,7)$. La infección por CMV puede no desarrollar ningún síntoma $y$, por ello, no es catalogado como una enfermedad ${ }^{(2,8)}$. En la población pediátrica, la ECMV ocurre en el 11-18\% y la ECMV-RG ocurre en menos del $2 \%$ de los casos. Varios factores contribuyen a la aparición de resistencia, como el serotipo receptor de alto riesgo ( $D+/ R$-), mayor exposición previa al ganciclovir e historia de hospitalización relacionada con ECMV ${ }^{(3,4)}$.

Nuestro paciente tenía un estado serológico D+/R-, lo que contribuyó al desarrollo de la resistencia. Es importante mencionar que la evolución de resistencia no siguió la cronología natural. Normalmente, esta se establece después de 14-21 días de tratamiento con ganciclovir y de falta de mejora clínica del paciente, seguido de la identificación del gen mutado que otorga la resistencia ${ }^{(1,6)}$. Sin embargo, este patrón solo fue evidente después de la tercera hospitalización a los 277 días postrasplante.

En el primer pico de la carga viral, la decisión clínica inicial fue restablecer el ganciclovir con las dosis administradas anteriormente y el valganciclovir oral después de la mejora clínica. A pesar de ello, la carga viral aumentó exponencialmente, lo que conduce al segundo pico $y$, posteriormente, a una resolución de la enfermedad con mejora clínica y de laboratorio debido a la administración de dosis aumentadas de ganciclovir y valganciclovir. El tercer pico de la carga viral generó preocupación, sin mejora clínica después de aumentar las dosis de ganciclovir. Por lo tanto, se sospechó de ECMV-RG, y se cambió a tratamiento con foscarnet. Debido a la estabilización clínica con ganciclovir en el primer episodio, la resistencia solo se sospechó después del tercer episodio.

El paciente presentó la enfermedad al cabo de 121 días postrasplante. De este modo, se cataloga como ECMV de inicio tardío, por manifestarse 100 días después de la cirugía. Esta condición está asociada a diferentes variables como residir en un área no metropolitana y presentar cardiomiopatía isquémica como la etiología de la insuficiencia cardiaca. A su vez, la mortalidad en este grupo está ligada al rechazo del tejido y una falla renal en el transcurso de la enfermedad ${ }^{(9)}$. El paciente residía en un área metropolitana y no poseía una cardiopatía isquémica como la causa de su trasplante. Por ello, a pesar de conocer estos factores de riesgo, la posibilidad de una presentación tardía de ECMV debe considerarse.

La ECMV-RG es una ocurrencia rara en la población pediátrica. Kim et al., a través de una observación de siete años, solo identificaron un caso posterior a trasplante cardiaco ${ }^{(10)}$. Asimismo, Li et al. encontró cuatro casos en un período de observación de 10 años, lo que condujo a una incidencia menor del $2 \%{ }^{\left({ }^{3}\right)}$. El presente reporte es el primer caso de ECMV-RG postrasplante cardiaco en Perú y, más específicamente, el primero en población pediátrica. Es notable mencionar que el paciente desarrolló esta condición a pesar de recibir tratamiento profiláctico antiviral estándar. Casquero et al. mencionan que la terapia profiláctica debería ser ajustada a las necesidades de la institución y del propio paciente, debido que esta no es efectiva para evitar la presencia de resistencia a ganciclovir (11). Por lo tanto, se sugiere que el médico siempre debe sospechar de esta enfermedad, incluso si todas las medidas preventivas fueron tomadas, e incluso si hay mejora clínica y de laboratorio, como ocurrió en nuestro caso. Además, se debe buscar la individualización de cada paciente, con el fin de ajustar las dosis de acuerdo a sus necesidades.

Entre las limitaciones del caso, se resalta que nuestra institución no posee el equipo de laboratorio para detectar la mutación responsable de la resistencia; por lo tanto, esta prueba no pudo realizarse. Sin embargo, nos basamos en la condición clínica para detectarla. Por otro lado, la obtención de foscarnet fue tediosa dado su falta de disponibilidad en el Perú, por lo que su administración al paciente tuvo una ligera demora.

Este es el primer reporte peruano de ECMV-RG postrasplante cardiaco, que además registró una presentación inusual de la resistencia y fue diagnosticado con las características clínicas. En adición, la resistencia al tratamiento convencional debe ser siempre considerada, aun cuando exista una inicial remisión de la sintomatología y normalización de la carga viral. Por lo tanto, todos los pacientes con un serotipo de alto riesgo deben ser tratados como fuente potencial para adquirir ECMV-RG, y realizar la prueba de secuenciación, con el fin de identificar la mutación específica. Finalmente, mostramos que la ECMVRG puede ser remitida incluso después de un tratamiento prolongado con ganciclovir y valganciclovir.

Consentimiento del paciente/apoderado: Los padres del paciente acordaron la publicación de este reporte, basado en la condición de su hijo.

Agradecimientos: Damos las gracias a todo el personal de salud del Instituto Cardiovascular Nacional, por apoyarnos y alentarnos en el desarrollo de este reporte de caso.

Contribuciones de autoría: CRC, GCK, SV, RVA y BVV, participaron en la concepción y diseño del artículo; recolección de resultados; redacción del artículo; revisión crítica del artículo y aprobación de la versión final. CRC facilitó el aporte de las imágenes y obtención de datos clínicos del paciente.

Financiamiento: Esta investigación no recibió ningún fondo monetario de alguna agencia pública, comercial o sin fines de lucro, para su realización.

Conflicto de Intereses: Los autores no tienen ningún conflicto de interés que declarar 


\section{REFERENCIAS BIBLIOGRÁFICAS}

1. Azevedo LS, Pierrotti LC, Abdala E, Costa SF, Strabelli TM, Campos SV, et al. Cytomegalovirus infection in transplant recipients. Clinics (Sao Paulo). 2015;70(7):515-23. doi: 10.6061/ clinics/2015(07)09.

2. Mendez-Eirin E, Paniagua-Martin MJ, Marzoa-Rivas R, Barge-Caballero E, Grille-Cancela Z, Canizares A, et al. Cumulative incidence of cytomegalovirus infection and disease after heart transplantation in the last decade: effect of preemptive therapy. Transplant Proc. 2012;44(9):2660-2. doi: 10.1016/j. transproceed.2012.09.035.

3. Li F, Kenyon KW, Kirby KA, Fishbein DP, Boeckh M, Limaye AP. Incidence and clinical features of ganciclovir-resistant cytomegalovirus disease in heart transplant recipients. Clin Infect Dis. 2007;45(4):439-47.

4. Groetzner J, Reichart B, Roemer U, Reichel S, Kozlik-Feldmann R, Tiete A, et al. Cardiac transplantation in pediatric patients: fifteen-year experience of a single center. Ann Thorac Surg. 2005;79(1):53-60.
5. Costanzo MR, Dipchand A, Starling R, Anderson A, Chan M, Desai S, et al. The International Society of Heart and Lung Transplantation Guidelines for the care of heart transplant recipients. J Heart Lung Transplant. 2010;29(8):914-56. doi: 10.1016/j.healun.2010.05.034.

6. Boivin G, Goyette N, Rollag H, Jardine AG, Pescovitz MD, Asberg A, et al. Cytomegalovirus resistance in solid organ transplant recipients treated with intravenous ganciclovir or oral valganciclovir. Antivir Ther. 2009;14(5):697-704.

7. Kotton CN, Kumar D, Caliendo AM, Asberg A, Chou S, Danziger-Isakov L, et al. Updated international consensus guidelines on the management of cytomegalovirus in solid-organ transplantation. Transplantation. 2013;96(4):333-60. doi: 10.1097/TP.0b013e31829df29d.

8. Roman A, Manito N, Campistol JM, Cuervas-Mons V, Almenar L, Arias M, et al. The impact of the prevention strategies on the indirect effects of CMV infection in solid organ transplant recipients. TransplantRev(Orlando).2014;28(2):8491. doi: 10.1016/j.trre.2014.01.001.
9. Santos CA, Brennan DC, Fraser VJ, Olsen MA. Incidence, risk factors, and outcomes of delayed-onset cytomegalovirus disease in a large, retrospective cohort of heart transplant recipients. Transplant Proc. 2014;46(10):3585-92. doi: 10.1016/j. transproceed.2014.08.043.

10. Kim YJ, Boeckh M, Cook L, Stempel $\mathrm{H}$, Jerome KR, Boucek R, Jr., et al. Cytomegalovirus infection and ganciclovir resistance caused by UL97 mutations in pediatric transplant recipients. Transpl Infect Dis. 2012;14(6):611-7. doi: 10.1111/j.1399-3062.2012.00760.x.

11. Casquero S, Rangel D, Lage E, Sobrino $\mathrm{M}$, Cristobo P, Cordero E, et al. Preemptive therapy in the prevention of cytomegalovirus disease in high- and lowrisk heart transplant recipients. Transplant Proc. 2012;44(7):2113-4. doi: 10.1016/j. transproceed.2012.07.066.

Correspondencia: Bryan Valcarcel Valdivia Dirección: Psje. Huamanga 216, Balconcillo, La Victoria. Lima, Perú

Teléfono: (+51) 947375871

Correo electrónico:bryan.valcarcel@gmail.com

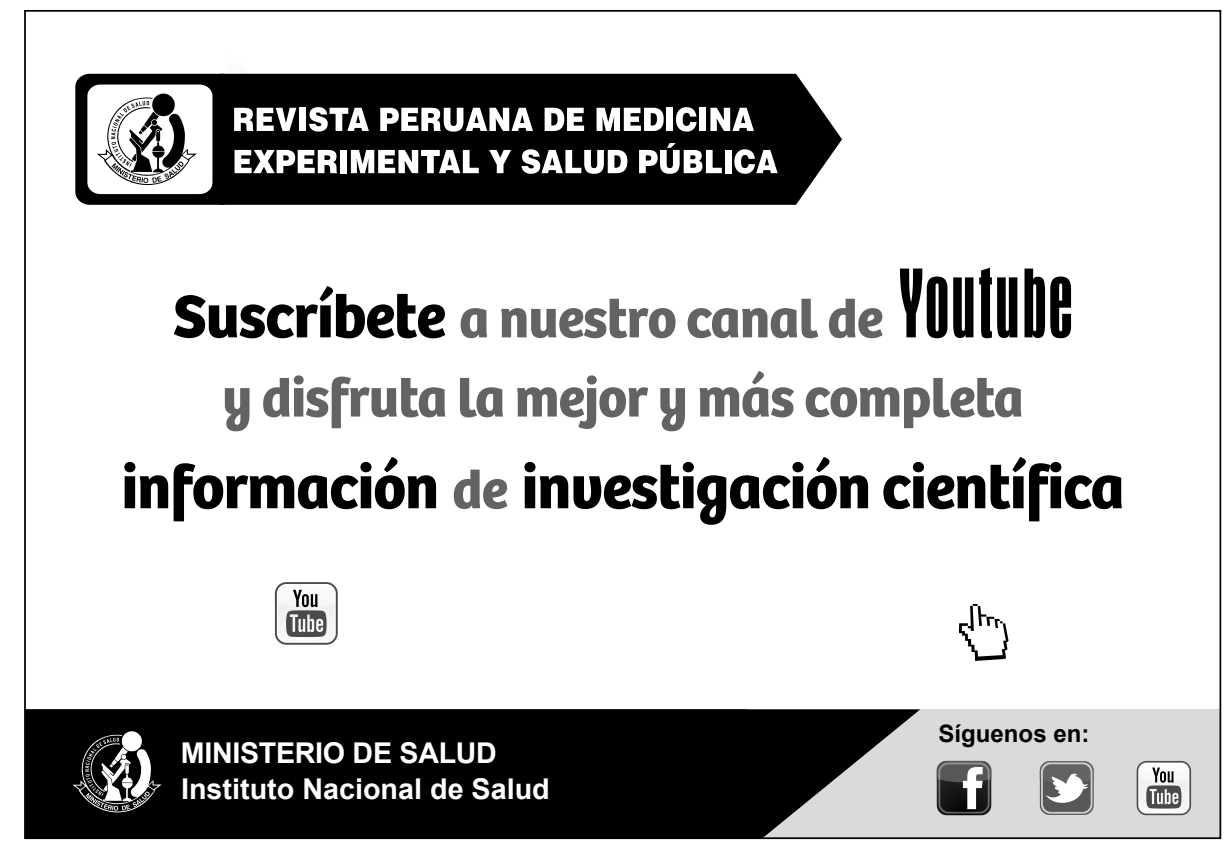

